\title{
LATIS3D: The Gold Standard for Laser-Tissue-Interaction Modeling
}

R.A. London, A.M. Makarewicz, B.-M. Kim, N.A. Gentile, T.-Y. B. Yang, M. Drlik, L. Vincent

\section{March 16, 2000}

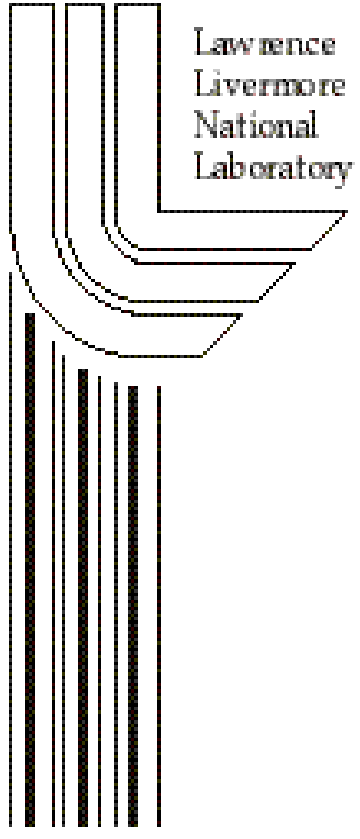




\section{DISCLAIMER}

This document was prepared as an account of work sponsored by an agency of the United States Government. Neither the United States Government nor the University of California nor any of their employees, makes any warranty, express or implied, or assumes any legal liability or responsibility for the accuracy, completeness, or usefulness of any information, apparatus, product, or process disclosed, or represents that its use would not infringe privately owned rights. Reference herein to any specific commercial product, process, or service by trade name, trademark, manufacturer, or otherwise, does not necessarily constitute or imply its endorsement, recommendation, or favoring by the United States Government or the University of California. The views and opinions of authors expressed herein do not necessarily state or reflect those of the United States Government or the University of California, and shall not be used for advertising or product endorsement purposes.

Work performed under the auspices of the U. S. Department of Energy by the University of California Lawrence Livermore National Laboratory under Contract W-7405-Eng-48.

This report has been reproduced

directly from the best available copy.

Available to DOE and DOE contractors from the

Office of Scientific and Technical Information

P.O. Box 62, Oak Ridge, TN 37831

Prices available from (423) 576-8401

http:/ /apollo.osti.gov/bridge/

Available to the public from the National Technical Information Service

U.S. Department of Commerce 5285 Port Royal Rd., Springfield, VA 22161

http://www.ntis.gov/

OR

Lawrence Livermore National Laboratory

Technical Information Department's Digital Library

http://www.llnl.gov/tid/Library.html 


\title{
LATIS3D: The Gold Standard for Laser-Tissue-Interaction Modeling
}

\author{
R. A. London, A. M. Makarewicz, B.-M. Kim, N. A. Gentile, T.-Y. B. Yang, M. Drlik \\ Lawrence Livermore National Laboratory, P. O. Box 808, Livermore California 94550 \\ and \\ L. Vincent \\ ScanSoft, Inc., 3400 Hillview Ave., Palo Alto, CA 94303
}

\begin{abstract}
The goal of this LDRD project has been to create LATIS3D-the world's premier computer program for laser-tissue interaction modeling. The development was based on recent experience with the 2D LATIS code and the ASCI code, KULL. With LATIS3D, important applications in laser medical therapy were researched including dynamical calculations of tissue emulsification and ablation, photothermal therapy, and photon transport for photodynamic therapy. This project also enhanced LLNL's core competency in laser-matter interactions and high-energy-density physics by pushing simulation codes into new parameter regimes and by attracting external expertise. This will benefit both existing LLNL programs such as ICF and SBSS and emerging programs in medical technology and other laser applications.
\end{abstract}

The purpose of this project was to develop and apply a computer program for laser-tissue interaction modeling to aid in the development of new instruments and procedures in laser medicine. The computer program is called LATIS3D, meaning lasertissue interaction in 3-dimensions. The development of LATIS3D has been accomplished by utilizing parts of existing computer programs at Lawrence Livermore National Laboratory and adding to them the special features needed for laser-tissue modeling.

Activities have focused on developing new algorithms, enhancing existing algorithms, developing a user interface, and developing a database of tissue physical properties. Numerous applications of laser tissue modeling were pursued, including dynamical models for tissue emulsification and ablation, thermal models for photothermal therapy, and photon transport models for photodynamic therapies.

The technical results of this project have largely been published in several journal articles and conference proceedings. These results will be summarized in the following paragraphs, with most details provided by the referenced publications. The most recent results are described in more detail since they have not been previously published.

The early phase of the project concentrated on enhancing algorithms in the LATIS computer program for future porting to LATIS3D. LATIS is a 2-dimensional program, which was largely developed prior to the current project. ${ }^{1}$ Enhancements were made to enable LATIS to model ultra-short pulse tissue ablation. This was applied towards the design of a laser-based system for ultra-high precision drilling of dental and other hard tissues. ${ }^{2}$ In addition, several theoretical studies were made of laser produced vapor 
bubbles in soft tissues. These studies required advances in the equations-of-state used for soft tissues and in the material strength and failure models in the hydrodynamic package. Such bubbles have been utilized to emulsify blood clots to treat strokes. An extended Rayleigh model was developed and applied to predicting bubble dynamics in various scenarios ${ }^{3}$. Detailed LATIS simulations in $1-\mathrm{D}^{4}$ and $2-\mathrm{D}^{5}$ symmetry were done and used to design a system for blood clot emulsification. A scaling model was developed for laser generated bubbles and comparisons made to LATIS simulations. ${ }^{6}$ A 2-D Rayleigh model was developed to provide fast calculations of bubble dynamics in the presence of spherical symmetry-breaking material objects, such as optical fibers and blood vessels. ${ }^{7}$ Other results included the development and application of material failure models for soft tissue removal via spallation, ${ }^{8,9}$ and the development and application of models for photothermal medical therapy. ${ }^{10,11}$

The later phase of the project focused on building from the KULL computer program to develop a 3-dimensional laser tissue interaction modeling capability. KULL is a new computer program being developed at LLNL primarily for laser fusion and high energy density experiments under the Advanced Strategic Computing Initiative. The specific components of KULL used for laser tissue modeling, currently the Monte Carlo photon transport package and the hydrodynamics package, are labeled LATIS3D.

Two laser medicine applications were addressed with LATIS3D. The first application was the study of optoacoustic stress waves. These waves have several uses in medicine including the destruction of blood clots, kidney stones and gall stones, skin regeneration, and the induction of cell permeability changes for drug delivery. We have modeled stress wave generation and propagation in an effort to optimize the design of instruments for these therapies. In particular, we have studied techniques to generate and focus the stress waves using optical fibers. The results of our simulations agree qualitatively with experimental data for both a flat-tipped fiber and a focusing lens. The results indicate that focusing tips at the ends of optical fibers will allow sharp focusing and enhance development of both compressive and tensile pressures. Preliminary results have been published in reference [12]. ${ }^{12}$

The other application was the design of laser protocols for photodynamic therapy (PDT) for treating arthritis. To address this problem, we need to add a laser source, tissue scattering, absorption and refractive processes to LATIS3D.

The first studies used simplified three-dimensional knee models composed of block shaped regions. We performed Monte-Carlo simulations of the distribution and absorption of light, examining the effect of source placement and the degree of inflammation of the synovial membrane (due to arthritis). ${ }^{13}$ This study showed that it is desirable to deliver light energy directly to the synovial membrane using an optical fiber rather than externally. It was also found that the diseased synovial membrane limits light penetration. We also examined the effect of the smoothness of tissue boundaries on the reflection and transmission of light, which depends strongly on angle at which the light hits the boundary between two materials. Calculations were performed using both a jagged boundary between the synovial fluid and bone tissue and a smooth, tilted boundary. The results show that with the jagged boundary the relative error in the light intensity can be as high as $25 \% .^{13}$ This implies that using meshes with smooth tissue surfaces will produce more accurate light distribution results.

To begin to address more realistic models we obtained a public domain MRI dataset of a human knee. Manual segmentation of a sample knee MRI (127 images at 1 $\mathrm{mm}$ resolution) was performed to provide a baseline image for comparison to future automated segmentation attempts and to provide input for laser light simulations. The knee image is from the Chapel Hill Volume Rendering Test Data Set and was obtained via anonymous ftp from the University of North Carolina Department of Computer Science, Chapel Hill, NC 27599-3175. Data was taken on the Siemens Magnetom and 
provided courtesy of Siemens Medical Systems, Inc., Iselin, NJ. Segmentation was performed using Mimics (Materialise, Ann Arbor, MI). Mimics allows some automation of the segmentation process, using thresholding, region growing, and cavity fill techniques. Significant manual refinement (pixel-by-pixel) editing, of the automated segmentation was required to refine the tissue boundaries to an accuracy appropriate for light propagation modeling. Approximately 100 man-hours of manual interaction was required to segment the femur, tibia, patella, fibula, muscle, skin, quadriceps tendon, patellar ligament, and the anterior and posterior cruciate ligaments. A volume rendering of the bones and muscles is shown in Figure 2. The skin, tendon, and ligaments have been omitted for clarity.

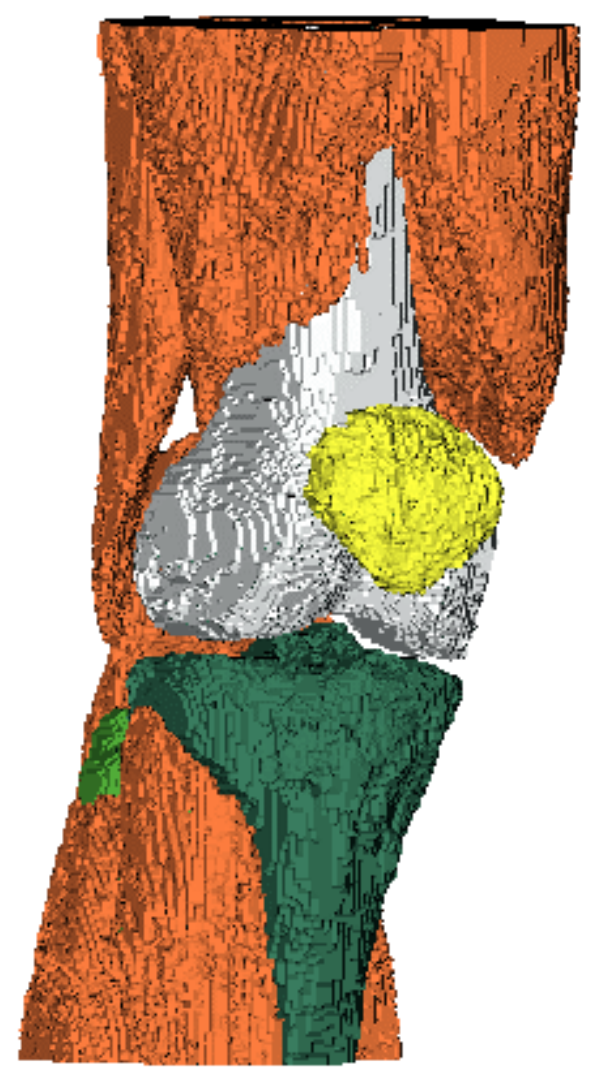

Figure 1. Volume rendering of the manual segmentation, performed using the commercial software Mimics (Materialise, Ann Arbor, MI). The femur, tibia, patella, fibula, and muscle are shown. The skin, tendons, and ligaments have been omitted for clarity.

Using the manual segmentation, we generated a regular voxel based mesh of an 8$\mathrm{cm}$-cubed region surrounding the contact surfaces of the femur and tibia. This was imported into LATIS3D and used for a light transport calculation, shown in Figure 2. Due to the lack of resolution in the MRI image and the fairly crude segmentation, only the femur and tibia were included in the calculation. The bones were manually separated in the vertical direction by $1 \mathrm{~cm}$ to model the situation of arthroscopic surgery, in which a fluid is injected into the joint to open up the joint region. The light source was a $4 \mathrm{~cm}$ long fiber length placed along the $\mathrm{x}$-axis $(\mathrm{z}=0, \mathrm{y}=0)$ extending from $\mathrm{x}=-2$ to $\mathrm{x}=2$. The 700-nm laser light was emitted in radial direction from the fiber. The absorption of light 
near the surface of the bones on either side of the joint fluid is evident in Fig. 2. These results demonstrate the capability to translate an anatomical model derived by segmentation into a mesh, which can be imported to the LATIS3D. The results also demonstrate the capability to perform a light transport calculation using the model. However, it is clear from the low resolution of the results and the omission of several important tissues, that this is but a preliminary result, which needs to be followed up with more refined models and simulations. Further investigations require the capability to obtain accurate 3-D, surface conforming meshes containing all of the tissues of interest, and to be able to obtain them in a more automated manner.

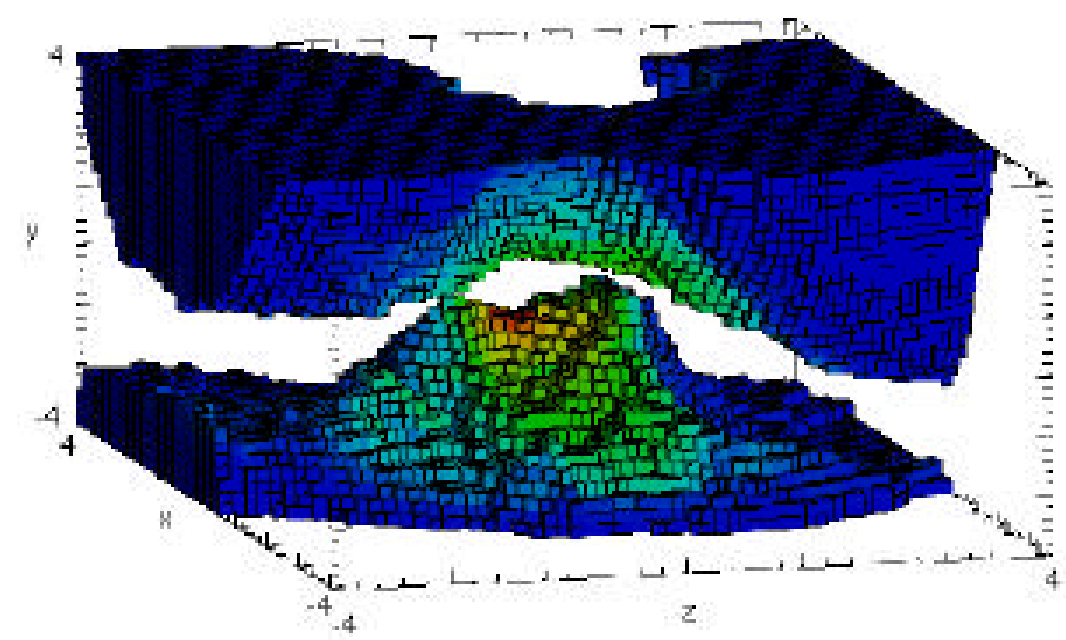

Figure 2. Laser light distribution at the center of the knee joint. The segmentation shown in Figure 1 was imported into LATIS3D for the calculation. Distances are in $\mathrm{cm}$. The gray scale goes from light gray (low light level) to black (high level). The regular voxel mesh lines at the surfaces of the femur and tibia are also shown.

In order to generate better geometrical models for 3-D tissue simulations and to speed-up their generation, we began to explore the use of advanced image segmentation methods. In the past 20 years a powerful new approach to image analysis, called the morphology method has been developed. ${ }^{14,15}$ Preliminary work was done to segment the public domain MRI dataset with morphological techniques. Results are shown in Figure 3. The segmentation was accomplished by first filtering of each slice was using nearest neighbors. The data was then processed in 2-D slices. A circular opening was applied to deepen the valleys surrounding the cancellous bones.

Thresholding of this opening provided a reasonable extraction of the bones, although at this point they were erroneously connected in small regions. Watershed segmentation was then applied to separate the bones to produce the boundaries shown in Figure 3. The morphological techniques used here were mostly two-dimensional, but can, in general, be adapted to three dimensions. This method was found to give results better than the manual segmentation, and more importantly, with a smaller amount of effort. 

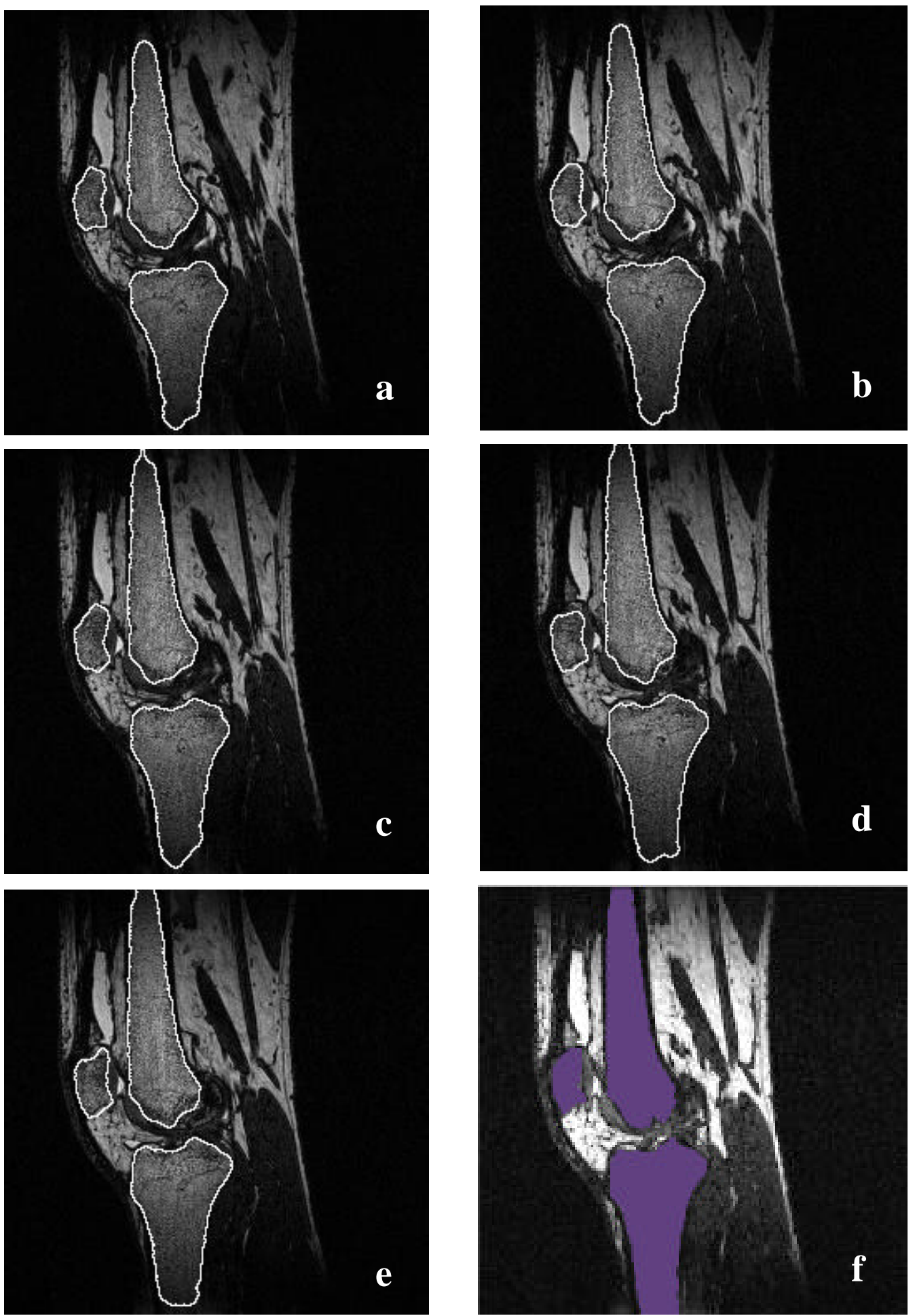

Figure 3: Serial MRI slices of a knee with $1 \mathrm{~mm}$ separation; (a-e) border of cancellous (spongy) bone as determined by our initial automated segmentation technique, utilizing $2 \mathrm{D}$ watershed methods; (f) segmentation results using Mimics (Materialise, Inc., Ann Arbor, MI) for the same slice as shown in (e), which utilizes some automation (thresholding, region growing, etc.) but requires significant manual refinement. 
Future work will extend the morphological methods to generate a semi-automatic computer aided segmentation capability. This will allow us to generate numerical meshes for performing 3-D simulations of laser light transport in with realistic tissue models. We will then pursue further applications in laser medicine, including designs for laser light delivery systems for photodynamic therapy, and detailed designs for optoacoustic lithotripsy devices.

This work was performed under the auspices of the U.S. Department of Energy by the University of California Lawrence Livermore National Laboratory under contract No. W-7405-Eng-48.

\section{References}

${ }^{1}$ R. A. London, M. E. Glinsky, G. B. Zimmerman, D. S. Bailey, D. C. Eder, and S. L. Jacques, "Laser-Tissue Interaction Modeling with LATIS," Applied Optics, 36, 9068 (1997).

${ }^{2}$. D. S. Bailey, R. A. London, and W. E. Alley, "Hard-Tissue Ablation Simulations Using the LATIS Computer Code", in Laser-Tissue Interaction IX, Proc. SPIE 3154, (Bellingham, 1998), p. 232.

${ }^{3}$ M. E. Glinsky, P.A. Amendt, D. S. Bailey, R. A. London, A. M. Rubenchik, and M. Strauss, "Extended Rayleigh Model of Bubble Evolution with Material Strength Compared to Detailed Dynamic Simulations," in Laser-Tissue Interaction VIII, Proc. SPIE 2975 (Bellingham, 1997) p. 318, and "An Extended Rayleigh Model of Bubble Evolution,” submitted to Phys. Fluids

${ }^{4}$ P. A. Amendt, M. Strauss, R. A. London, M. E. Glinsky, D. J. Maitland, P. M. Celliers, S. R. Visuri, D. S. Bailey, D. A. Young, and D. Ho, "Modeling of Bubble Dynamics in Relation to Medical Applications," in Laser-Tissue Interaction VIII, Proc. SPIE 2975 (Bellingham, 1997) p. 362, and "Laserinitiated Bubble Evolution for Medical Applications: Dynamics and Energetics," submitted to J. App. Phys.

${ }^{5}$ P. Amendt, R. A. London, M. Strauss, M. E. Glinsky, D. J. Maitland, P. M. Celliers, S. R. Visuri, D. S. Bailey, D. A. Young, D. Ho., C. P. Lin, and M. W. Kelley, "Simulation Studies of Vapor Bubble-Generation by Short-Pulse Lasers," in Laser-Tissue Interaction, Tissue Optics, and Laser Welding III, Proc. SPIE 3195 (Bellingham, 1998), p. 259.

${ }^{6}$ R. A. London, D. S. Bailey, P. A. Amendt, S. R. Visuri, and V. C. Esch, "Scaling model for laser-produced bubbles in soft tissue," in Laser-Tissue Interaction IX, Proc. SPIE 3154, (Bellingham, 1998), p. 256.

${ }^{7}$ M. Strauss, M. Friedman, E. Gurewitz, P. Amendt, R. A. London and M. E. Glinsky, "Two-Dimensional Rayleigh Model of Vapor Bubble Evolution," in Laser-Tissue Interaction X, Proc. SPIE 3601, (Bellingham, 1999) p. 212.

${ }^{8}$ M. E. Glinsky, D. S. Bailey, and R. A. London, "LATIS Modeling of Laser Induced Midplane and Backplane Spallation," in Laser-Tissue Interaction VIII, Proc. SPIE 2975, (Bellingham, 1997), p. 374.

${ }^{9}$ M. Strauss, Y. Kaufman, M. Sapir, P. Amendt, R. A. London and M. E. Glinsky, "Self-Consistent Evolution of Tissue Damage Under Stress Wave 
Propagation," in Laser-Tissue Interaction X, Proc. SPIE 3601, (Bellingham, 1999) p. 178.

${ }^{10}$ R. A. London, V. Esch, and D. Papademetriou "Numerical Simulations of a Diode Laser BPH Treatment System," in Laser-Tissue Interaction X, Proc. SPIE 3601, (Bellingham, 1999) p. 141.

${ }^{11}$ R. A. London, J. Eichler, J. Liebetruth, and L. Ziegenhagen, "Design of a Protocol for Combined Laser Hyperthermia-Photodynamic Therapy in the Esophagus," to appear in Lasers in Surgery: Advanced Characterization, Therapeutics, and Systems X, Proc. SPIE 3907, (Bellingham, 2000), and J. Eichler, J. Liebetruth, R. A. London, and L. Ziegenhagen, "Temperature Distribution for Combined Laser HyperthermiaPhotodynamic Therapy in the Esophagus," to appear in Medical Engineering and Physics, 2000.

${ }^{12}$ A. J. Makarewicz, S. R. Visuri, R. A. London, D. S. Miller, "Modeling Optically Generated Focused Shockwaves," in Laser-Tissue Interaction X, Proc. SPIE 3601, (Bellingham, 1999) p. 207.

${ }^{13}$ B.-M. Kim, N. A. Gentile, K. B. Trauner, and R. A. London, "Three Dimensional Monte Carlo Modeling of Laser-Tissue Interaction," in LaserTissue Interaction X, Proc. SPIE 3601, (Bellingham, 1999) p. 320.

${ }^{14}$ J. Serra, "Image Analysis and Mathematical Morphology," (Academic Press, London 1988),

${ }^{15}$ L. Vincent and P. Soille, "Watersheds in Digital Spaces: an Efficient Algorithm Based on Immersion Simulations," IEEE Transactions on Pattern Analysis and Machine Intelligence 13, 583 (1991). 